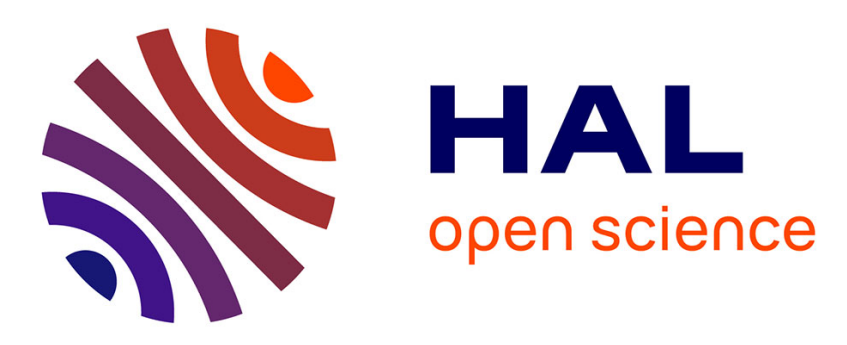

\title{
Measuring Interfacial Polymerization Kinetics Using Microfluidic Interferometry
}

Arash Nowbahar, Vincent Mansard, Jodi Mecca, Mou Paul, Tina Arrowood, Todd Squires

\section{> To cite this version:}

Arash Nowbahar, Vincent Mansard, Jodi Mecca, Mou Paul, Tina Arrowood, et al.. Measuring Interfacial Polymerization Kinetics Using Microfluidic Interferometry. Journal of the American Chemical Society, 2018, 140 (9), pp.3173-3176. 10.1021/jacs.7b12121 . hal-01734561

\section{HAL Id: hal-01734561 \\ https://hal.science/hal-01734561}

Submitted on 14 Mar 2018

HAL is a multi-disciplinary open access archive for the deposit and dissemination of scientific research documents, whether they are published or not. The documents may come from teaching and research institutions in France or abroad, or from public or private research centers.
L'archive ouverte pluridisciplinaire HAL, est destinée au dépôt et à la diffusion de documents scientifiques de niveau recherche, publiés ou non, émanant des établissements d'enseignement et de recherche français ou étrangers, des laboratoires publics ou privés. 


\section{Measuring Interfacial Polymerization Kinetics Using Microfluidic Interferometry \\ Arash Nowbahar, ${ }^{\dagger}$ Vincent Mansard, ${ }^{\ddagger}$ Jodi M. Mecca, ${ }^{\llbracket}$ Mou Paul,, Tina Arrowood, $^{\S}$ and Todd \\ M. Squires*, \\ $\dagger$ Department of Chemical Engineering, University of California Santa Barbara, CA 93106 \\ $\ddagger$ Laboratory for Analysis and Architecture of Systems (LAAS-CNRS) Toulouse, 31400 Toulouse, France \\ ๆFormulation Science, Core Research and Development, Dow Chemical Company, Midland, MI 48674 \\ $\S$ Dow Water \& Process Solutions, Dow Chemical Company, Edina, MN 55439}

Received January 29, 2018; E-mail: squires@engineering.ucsb.edu

\begin{abstract}
Interfacial polymerization is used in a range of fields, both academic and industrial, for the production of fibers, capsules, and films. Despite its widespread use, measuring the reaction kinetics of interfacial polymerization has remained a challenge. For example, reaction kinetics for polyamide reverse osmosis membranes are difficult to obtain and rarely reported due to the thinness of films, and rapidity of their formation at the liquid-liquid interface. Here, polyamide film formation is studied using a microfluidic, interferometry-based technique to measure monomer concentration near the interface as the reaction occurs. Our results are consistent with a polymerization reaction that is initially controlled by a reaction-diffusion boundary layer within the organic phase. Using simple scaling arguments to analyze our data, we report the first measurements of the reaction rate constant for this system.
\end{abstract}

Reacting monomers at an interface between two immiscible solvents is a robust and common method for producing fibers, ${ }^{1,2}$ capsules, ${ }^{3,4}$ and ultrathin films. ${ }^{5}$ While interfacial polymerization has been known for over 50 years $^{2}$ and is used in large-scale manufacturing processes today, ${ }^{6}$ in situ techniques to measure interfacial reaction kinetics are limited, system-specific, and often cannot capture the dynamics of fast reactions.

In particular, polyamide reverse osmosis (RO) membranes are made through interfacial polymerization, whose reaction kinetics have remained notoriously difficult to measure. The difficulty arises from the rapid ( $\sim$ seconds $)^{2}$ formation of a thin $(\sim 100 \mathrm{~nm})^{6}$ film at the liquid-liquid interface. In addition, multiple phenomena are coupled in this process: chemical reactions, solute transport between phases, and polymer phase separation, adding further complexity to the problem. ${ }^{7,8}$ For example, the reaction between m-phenylenediamine (MPD) and trimesoyl chloride (TMC) monomers, commonly used in RO membrane synthesis (Fig. 1a), ${ }^{9}$ is believed to occur within the oil phase. MPD must therefore partition from the water to the oil, diffuse within the oil, and react with TMC to form oligomers. ${ }^{2}$ As the oligomers lengthen, phase separation occurs, growing a polymer film that impedes the further transport of amine on one side of the film to the acid chloride on the other. ${ }^{10,11}$ The reaction thus slows, and becomes 'self-limiting'. ${ }^{11}$ While there is broad agreement regarding this mechanism for RO membrane formation, most details remain unknown or poorly characterized, including the intrinsic reaction rate constants, and the effects of the physical and chemical properties of the solutions themselves (e.g diffusivities, partition coefficients,
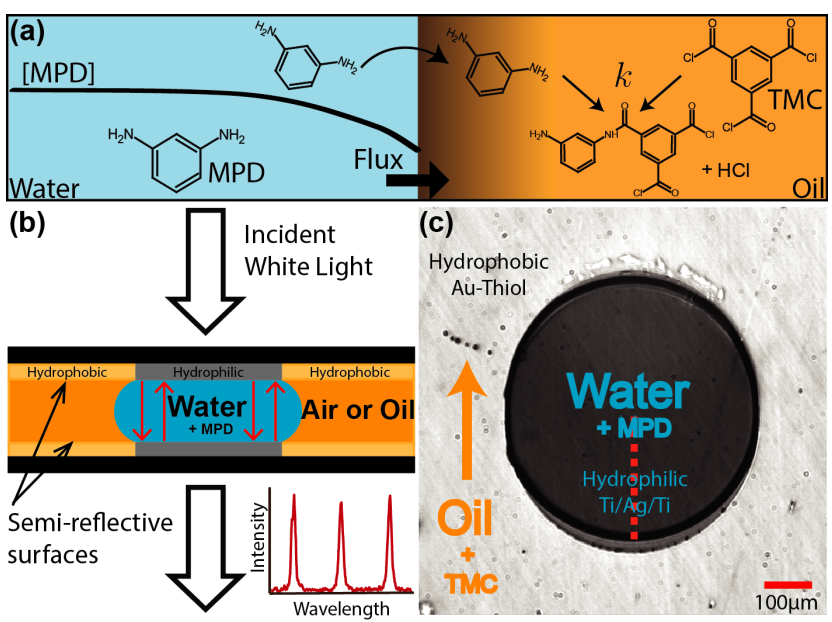

Figure 1. (a) Structures of MPD and TMC in the aqueous and oil phase respectively. MPD partitions into the oil and reacts with TMC, reducing the concentration of MPD in the aqueous phase near the interface. Flux into the oil phase is measured with interferometry. (b) Side view of microfluidic device for interferometry. Photo-patterned hydrophilic region traps aqueous phase. Drop profile shown is exaggerated, as the channel height is a tenth of the drop radius. Light entering from above reflects between surfaces, with different wavelengths interfering differently. Spectrometer splits the transmitted light from each pixel into a series of peaks at wavelengths that interfere constructively. Changes in these peak wavelengths correspond to changes in local refractive index. (c) Top view of trapped water drop with arrow indicating the direction oil flows to initiate the reaction. Light is collected along a slit (red dash), to resolve refractive index profiles of an MPD solution as they evolve in space and time.

concentrations) on the overall kinetics. ${ }^{12}$

Attempts to measure such interfacial polymerization kinetics often involve arresting the polymerization at various times and measuring membrane mass or thickness, or sampling solution concentrations with time. ${ }^{8,11,13-21}$ Such methods, however, cannot capture the dynamics of fast-forming films. Dynamic measurements of solution $\mathrm{pH}$ have captured monomer consumption in polyurea encapsulation, ${ }^{3,22-24}$ and have recently been applied to polyamide formation. ${ }^{25}$ These techniques inherently probe properties of bulk solutions, rather than conditions at the interface where the reaction occurs. Solute transport effects are therefore lumped in with kinetic processes, and measurements may conflate the two. The growth dynamics of polyamide membranes have additionally been studied through pendant drop tensiometry, light reflectometry, ${ }^{11}$ and diffuse reflectance spectroscopy. ${ }^{26}$ These methods capture qualitative features of the reaction, 

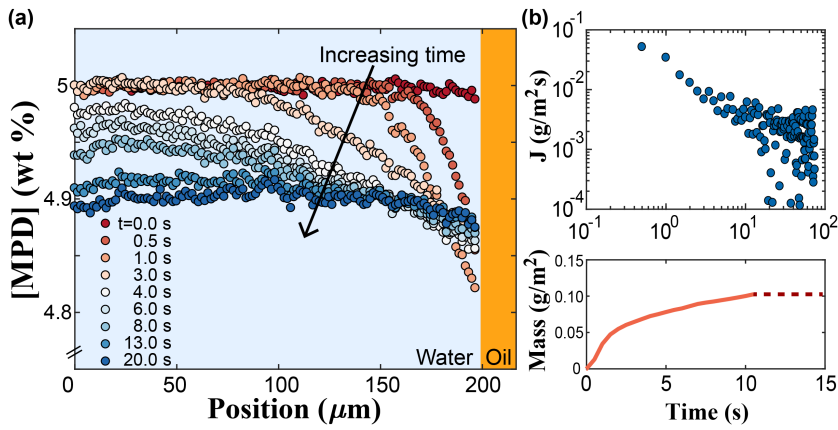

Figure 2. (a) Concentration profiles measured along the drop radius (drop center is at the origin) for the reaction between $5 \mathrm{wt} \%$ MPD and $0.5 \mathrm{wt} \%$ TMC. (b) Flux of MPD leaving the aqueous phase (top), and the total mass of MPD that has left the drop (bottom) for the experiment shown in (a). The flux is integrated (to find the mass) until fluctuations become larger than the flux itself, which occurs at approximately $10 \mathrm{~s}$ (Fig. S4)

revealing that more than half of the film forms within the first few seconds, and allows the growth rate to be estimated. However, relating measurements of interfacial tension and light reflection to monomer consumption or polymer production is difficult. As others have noted, ${ }^{23,24}$ there is a continuing need for a systematic experimental study of interfacial polymerization kinetics.

In this work, we present a new method to probe interfacial reaction kinetics. We design microfluidic interferometry devices that track monomer concentration profiles as they evolve in space and time. Specifically, the flux of amine leaving the aqueous phase is determined from spatial and temporal measurements of amine concentration near the oil/water interface. Relating this flux to the reaction that consumes it provides unprecedented measurements of reaction kinetics, and therefore new insights into the polymerization process of MPD with TMC. At the start of the interfacial polymerization process, monomer-monomer reactions occur within a reaction-diffusion boundary layer, and thus follow simple scalings. Our measurements provide, for the first time, the reaction rate constant of the MPD-TMC interfacial polymerization reaction.

Our technique employs multiple-beam interferometry ${ }^{27,28}$ in a microfluidic device ${ }^{29}$ to measure changes in refractive index as monomer is consumed by the reaction. Briefly, white light sent through two semi-reflective surfaces constructively interferes at wavelengths that depend upon the optical path (the distance separating the two mirrors multiplied by the refractive index of the solution). These wavelengths appear as discrete peaks when the transmitted light is split by a spectrometer (Fig. 1b). Changes in concentration cause changes in refractive index, which causes peak wavelengths to shift. Spatial resolution is obtained by collecting light through a slit, and tracking the refractive index for each pixel within the slit, therefore being set by the objective used (here 0.78 $\mu \mathrm{m} /$ pixel). By relating refractive index changes with concentration changes (Fig. S1), monomer concentration can be monitored spatially and temporally.

Employing this technique to study an interfacial reaction requires creating a stable oil-water interface. To this aim, we developed mirrored, hydrophobic, microfluidic devices, with photo-patterned $500 \mu \mathrm{m}$-diameter circular hydrophilic spots, as shown in Fig. 1b-c (see SI for details). ${ }^{30}$ Filling the device with aqueous MPD solution, then displacing the solution with air creates a pancake-shaped MPD drop that wets the hydrophilic spot, pins the water-air contact line, and holds the drop in place. The experiment begins when a solution of TMC in decane is pushed into the channel and then stopped, initiating the interfacial polymerization reaction. A slit of light is collected at the leading edge ("upstream") of the drop, and MPD concentration profile is tracked in space and time as the reaction proceeds (Fig. 1c).

At the moment of oil contact, the MPD concentration $[M P D]$ near the interface drops, as MPD diffuses into the oil phase and begins to react (Fig. 2a). Within the first few seconds, the interfacial MPD concentration begins to rise, indicating a slowing reaction as MPD is replenished from the bulk of the drop. With time, diffusion smooths out gradients in concentration, and the profiles flatten. Measured concentration profiles are indeed consistent with solutions to the diffusion equation, with the interfacial boundary condition set by the measured concentration (Fig. S2).

Measuring the slope of the concentration profiles at the interface gives the diffusive flux, $J$, of MPD into the oil,

$$
J=-\left.D \frac{\partial[M P D]}{\partial r}\right|_{r=R},
$$

where $D=840 \mu^{2} / \mathrm{s}$ is the measured diffusivity of MPD in water (see SI), $r$ is the radial coordinate, and $\mathrm{R}$ is the drop radius (Fig. 2b). The flux starts at a maximum, and decays within the first second as a power-law in time with exponent between $-\frac{1}{2}$ and $-\frac{3}{2}$, depending on starting concentrations (Fig. S5). This flux indicates the amount of amine leaving the aqueous phase with time. Integrating this flux over time reveals the total amount of amine that has reacted (Fig. 2b). Most of the mass of the membrane is formed within the first few seconds, consistent with other reports. ${ }^{26}$ Simple estimates further confirm these measurements to be reasonable: a measured MPD mass of $0.1 \mathrm{~g} / \mathrm{m}^{2}$ corresponds to a membrane thickness of $\sim 250 \mathrm{~nm}$ (density of $1.24 \mathrm{~g} / \mathrm{cm}^{3,31}$ with a ratio of $1 \mathrm{MPD}$ to $1 \mathrm{TMC}$ ), consistent with typical membrane thicknesses. ${ }^{6,11,32}$

These measurements provide direct access to the rate of monomer consumption as the polymerization occurs. A model is required to relate $J$ to properties of the reaction. Following Ref. 33, we consider the reaction to occur in two stages: monomer-monomer coupling in a reaction-diffusion boundary layer (of Danckwerts type ${ }^{34}$ ), followed by precipitation and film formation. We begin with the former, which holds only at the very early stage of the reaction.

At the instant the two phases come into contact, MPD diffuses into the oil, where it reacts homogeneously with TMC (assumed in excess) at a rate $6 k[M P D]_{o i l}[T M C]_{0}$, where $k$ is the reaction rate constant, $[M P D]_{\text {oil }}$ is the concentration of MPD in the oil, and $[T M C]_{0}$ is the starting TMC concentration. The factor 6 reflects bi-functional MPD and tri-functional TMC. Statistically, each amine monomer reacts within a time $\tau \sim \frac{1}{6 k[T M C]_{0}}$, after which it is consumed. During this time, the amine diffuses a distance $\delta \sim \sqrt{D_{o} \tau}$ from the interface, where $D_{o}$ is the MPD diffusivity in oil. A reaction-diffusion boundary layer of thickness

$$
\delta \sim \sqrt{\frac{D_{o}}{6 k[T M C]_{0}}}
$$

thus forms (Fig. S7). We assume the reaction-diffusion boundary layer to evolve quasi-steadily, since it develops faster than concentrations change appreciably within the aqueous drop. The flux in the water (eq. 1) balances the 
(a)

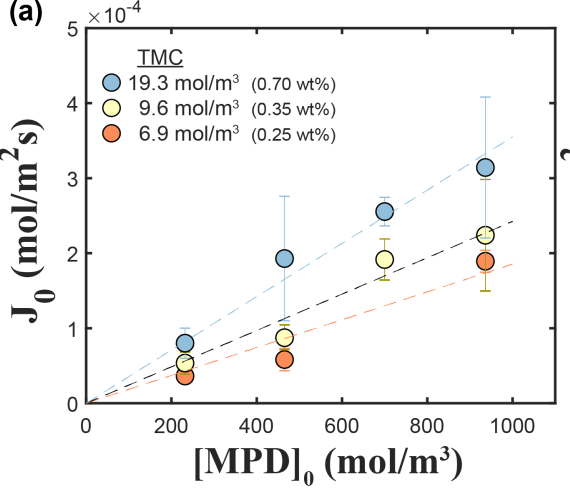

(b)

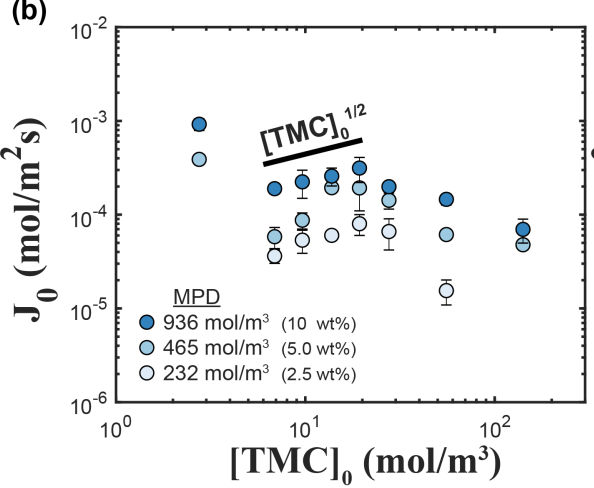

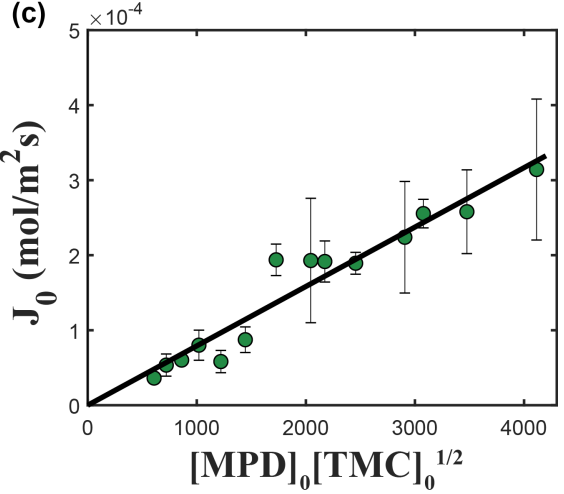

Figure 3. (a) Flux at the start of the reaction for fixed initial TMC concentrations, but varying initial MPD concentrations. $J_{0}$ scales linearly with $[M P D]_{0}$ as predicted. (b) Flux at the start of the reaction for fixed $[M P D]_{0}$, but varying $[T M C]_{0}$. $J_{0}$ scales linearly with $\sqrt{[T M C]_{0}}$ for a range of starting concentrations. (c) Plotting $J_{0}$ against $[M P D]_{0} \sqrt{[T M C]_{0}}$ (units of $\mathrm{mol}^{3 / 2} \mathrm{~m}^{-9 / 2}$ ), in $[T M C]_{0} \mathrm{range}$ of $6.8-19.3 \mathrm{~mol} / \mathrm{m}^{3}$, reveals a line with a slope of $7.9 \pm 0.6 \times 10^{-8} \mathrm{~m}^{5 / 2} \mathrm{~mol}^{-1 / 2} \mathrm{~s}^{-1}$. Error bars show standard error from at least three experiments for each point. Experiments are performed at room temperature (temperature deviations up to $\pm 3 \mathrm{~K}$, a possible source of the error).

flux in the oil across the boundary layer, and is given approximately by

$$
J_{0} \sim \frac{K D_{o}[M P D]_{0}}{\delta}
$$

where $K$ is the oil/water partition coefficient and $[M P D]_{0}$ the initial MPD concentration in the aqueous phase. Combining (2) with (3) gives

$$
J_{0} \sim K[M P D]_{0} \sqrt{6 k[T M C]_{0} D_{o}} .
$$

Thus at the very start, when the polymerization is dominated by bimolecular reactions between monomers, we predict the amine flux out of the drop to scale linearly with $[M P D]_{0}$ and $\sqrt{[T M C]_{0}}$.

To test this assertion, we run this reaction with various combinations of starting MPD and TMC concentrations, and measure the flux for each reaction, as in Fig. 2b. Figure $3 a-b$ reports the flux at the start of the reaction (the first data point in Fig. 2b) plotted against starting MPD and TMC concentrations. At fixed TMC concentrations, the initial reactive flux indeed scales linearly with starting MPD concentration (Fig. 3a, Fig. S6). Varying $[T M C]_{0}$ reveals a range of concentrations $\left(6.8-19.3 \mathrm{~mol} / \mathrm{m}^{3}\right)$ whose flux are consistent with the scaling proposed in eq. $4, \sim \sqrt{[T M C]_{0}}$, for a reaction-diffusion boundary layer (Fig. $3 \mathrm{~b}$ ).

We expect this scaling to hold as long as the starting assumptions hold. For example, the scaling should break down if $[T M C]_{0}$ is too low to be considered in excess. A crude estimate requires $[T M C]_{0}$ must at least exceed $[M P D]_{o i l, 0}$, the concentration of MPD in the oil. Requiring $K[M P D]_{0}=[M P D]_{o i l, 0}<[T M C]_{0}$, corresponding to $[T M C]_{0}>2 \mathrm{mM}(K=0.0024$, Fig. $\mathrm{S} 10)$ for these experiments, suggests the scaling should break down for $[T M C]_{0}$ $<5 \mathrm{mM}$. Deviations from this scaling at very high TMC concentrations $(>27.6 \mathrm{mM})$, may reflect $\mathrm{HCl}$ production from polyamide formation or TMC hydrolysis.

Over a range of concentrations, the starting flux is indeed consistent with scaling (4). We can thus determine the fundamental, monomer-monomer reaction rate constant $k$, from the boundary layer scaling, up to a numerical prefactor. A more accurate expression can be determined using finite element computations to model the reaction in this specific geometry (Fig. S8), revealing the prefactor to be approximately $1 \pm 0.2$. Figure $3 \mathrm{c}$ plots the starting flux against
$[M P D]_{0}[T M C]_{0}^{1 / 2}$, giving a straight line with slope $7.9 \pm$ $0.6 \times 10^{-8} \mathrm{~m}^{5 / 2} \mathrm{~mol}^{-1 / 2} \mathrm{~s}^{-1}$. Using this with $K=0.0024$ $\pm 2 \times 10^{-4}$ (Fig. S10), and an oil-phase MPD diffusivity $D_{o}=1630 \pm 90 \mathrm{\mu m}^{2} / \mathrm{s}$ (Fig. S11), in eq. (4) reveals a reaction rate constant $k=110 \pm 35 \mathrm{~L} / \mathrm{mol} \mathrm{s}$. This compares well with the rate constant measured for the analogous reaction between aniline and benzoyl chloride (mono-functional versions of monomers used here) in N-methyl-2-pyrrolidone, for which $k=128-164 \mathrm{~L} / \mathrm{mol}$ s. ${ }^{35,36}$ Given that N-methyl2-pyrrolidone is a more polar solvent which stabilizes the charged transition state, the slightly smaller value reported here for $k$ seems reasonable. ${ }^{37}$

The previous discussion holds for the early stage in the reaction, where transport resistance due to a dense film is absent. Figure 2b, and its analog for other conditions, shows the amine flux to decay as a power-law in time, with exponent between $-\frac{1}{2}$ and $-\frac{3}{2}$, after the first second. One would expect $J \sim t^{-\frac{1}{2}}$ if MPD diffused through a growing membrane with constant diffusivity $D_{m} \cdot{ }^{15}$ Amine fluxes that decay more rapidly than $t^{-\frac{1}{2}}$ would therefore suggest MPD diffusivities that decrease as the polymer network forms. This hypothesis has been proposed elsewhere,${ }^{33,38}$ and is consistent with the experimentally observed 'self-limiting' behavior. ${ }^{8,11}$ We leave a more detailed analysis of this phenomenon for further studies.

To summarize, we have demonstrated a microfluidic interferometry technique that provides a window into the rapid dynamics of an interfacial polymerization reaction of widespread interest. Measured concentration changes near the interface capture the dynamics of this interfacial polymerization, and simple scaling arguments describe monomer consumption at the start of the reaction. Such insights will ultimately aid in connecting input chemistry with observed structure, morphology, and RO performance, and in the design of desired ultrathin films. While we have specifically focused on polyamide RO membrane formation, the general methodology should be applicable to other interfacial reactions.

Acknowledgement This work was supported by the Dow Chemical Company through the Dow Materials Institute at the University of California, Santa Barbara (UCSB) with additional support from the National Science Foundation Graduate Research Fellowship Program under Grant 
DGE-1144085. A portion of this work was performed in the UCSB Nanofabrication Facility, part of the NSF-funded NNIN network, in the Microfluidics Laboratory within the California NanoSystems Institute, supported by UCSB and the UC, Office of the President, and the Shared Experimental Facilities of the Materials Research Science and Engineering Center at UCSB (MRSEC NSF DMR 1720256). We thank Douglas Vogus, Anirudha Banerjee, and Sarah Grazul for aid in measuring $K$, and Yanxian Lin for assistance with PFG NMR.

\section{Supporting Information Avail- able}

- SI: Experimental details. Numerical Computation of system.

- Drop Formation: Video of formation of aqueous air interface.

- Sending in Oil: Video of oil contacting aqueous drop.

- Concentration Profiles: Video showing concentration profiles developing with time.

\section{References}

(1) Huang, J.; Kaner, R. B. Journal of the American Chemical Society 2004, 126, 851-855.

(2) Morgan, P. W.; Kwolek, S. L. 1959, XL, 531-559.

(3) Yadav, S. K.; Khilar, K. C.; Suresh, A. K. AIChE Journal 1996, 42, 2616-2626.

(4) Polenz, I.; Brosseau, Q.; Baret, J.-C. Soft Matter 2015, 11, 2916-2923.

(5) Raaijmakers, M. J. T.; Benes, N. E. Progress in Polymer Science 2016, 63, 86-142.

(6) Petersen, R. J. Journal of Membrane Science 1993, 83, 81150.

(7) Berezkin, A. V.; Khokhlov, A. R. Journal of Polymer Science Part B: Polymer physics 2006, 44, 2698-2724.

(8) Ji, J.; Mehta, M. Journal of Membrane Science 2001, 192, $41-54$.

(9) Lee, K. P.; Arnot, T. C.; Mattia, D. Journal of Membrane Science 2011, 370, 1-22.

(10) Sundet, S. A. Journal of Membrane Science 1993, 76, 175-183.

(11) Chai, G.-Y.; Krantz, W. B. Journal of Membrane Science 1994, 93, 175-192.

(12) Karode, S.; Kulkarni, S.; Suresh, A.; Mashelkar, R. Chemical Engineering Science 1998, 53, 2649-2663.

(13) Song, Y.; Sun, P.; Henry, L. L.; Sun, B. Journal of Membrane Science 2005, 251, 67-79.

(14) Janssen, L. J. J. M.; Te Nijenhuis, K. Journal of Membrane Science 1992, 65, 59-68.

(15) Enkelmann, V.; Wegner, G. American Chemical Society, Polymer Preprints, Division of Polymer Chemistry 1975, 16, 409413.

(16) Wang, C.-y.; Wang, D.-c.; Chiu, W.-y.; Chen, L.-w. Angewandte Makromolekulare Chemie 1997, 248, 123-137.

(17) Tsai, H.-b.; Lee, Y.-d.; Hua, N. T. Journal of Polymer Science, Part A: Polymer Chemistry 1987, 25, 2195-2206.

(18) Bouchemal, K.; Couenne, F.; Briançon, S.; Fessi, H.; Tayakout, M. AIChE Journal 2006, 52, 2161-2171.

(19) Zhang, Q.; Shi, Y.; Zhan, X.; Chen, F. Colloids and Surfaces A Physicochemical and Engineering Aspects 2012, 393, 17-26.

(20) Gaudin, F.; Sintes-zydowicz, N. Colloids and Surfaces A : Physicochemical and Engineering Aspects 2012, 415, 328-342.

(21) Bradbury, J. H.; Crawford, P. J. Transactions of the Faraday Society 1967, V, 185-191.

(22) Yadav, S. K.; Suresh, A. K.; Khilar, K. C. AIChE Journal 1990, 36, 431-438.

(23) Wagh, S. J.; Dhumal, S. S.; Suresh, A. K. Journal of Membrane Science 2009, 328, 246-256.

(24) Dhumal, S. S.; Suresh, a. K. Polymer 2010, 51, 1176-1190.

(25) Behera, S.; Suresh, A. K. Polymer (United Kingdom) 2017, 127, 28-44.

(26) Matthews, T. D.; Yan, H.; Cahill, D. G.; Coronell, O.; Mariñas, B. J. Journal of Membrane Science 2013, 429, 71-80.

(27) Hariharan, P. Basics of Interferometry, 2nd ed.; Elsevier Inc.: Burlington, MA, 2007.
(28) Israelachvili, J. N. J.Colloid Interface Sci. 1973, 44, 259.

(29) Vogus, D. R.; Mansard, V.; Rapp, M. V.; Squires, T. M. Lab Chip 2015, 15, 1689-1696.

(30) Zhao, B.; Viernes, N. O.; Moore, J. S.; Beebe, D. J. Journal of the American Chemical Society 2002, 124, 5284-5285.

(31) Zhang, X.; Cahill, D. G.; Coronell, O.; Mariñas, B. J. Journal of Membrane Science 2009, 331, 143-151.

(32) Rao, A. P.; Joshi, S. V.; Trivedi, J. J.; Devmurari, C. V.; Shah, V. J. Journal of Membrane Science 2003, 211, 13-24.

(33) Freger, V. Langmuir 2005, 21, 1884-1894.

(34) Danckwerts, P. V. Transactions of the Faraday Society 1950, 46,300 .

(35) Wang, P.; Zhang, J.; Wang, K.; Luo, G.; Xie, P. Industrial \&s Engineering Chemistry Research 2016, 55, 6310-6316.

(36) Wang, P.; Wang, K.; Zhang, J.; Luo, G. AIChE Journal 2015 $61,3804-3811$

(37) Reichardt, C.; Welton, T. Solvents and Solvent Effects in Organic Chemistry, 4th ed.; Wiley-VCH Verlag, 2010.

(38) Freger, V.; Srebnik, S. Journal of Applied Polymer Science 2003, $88,1162-1169$. 
Graphical TOC Entry

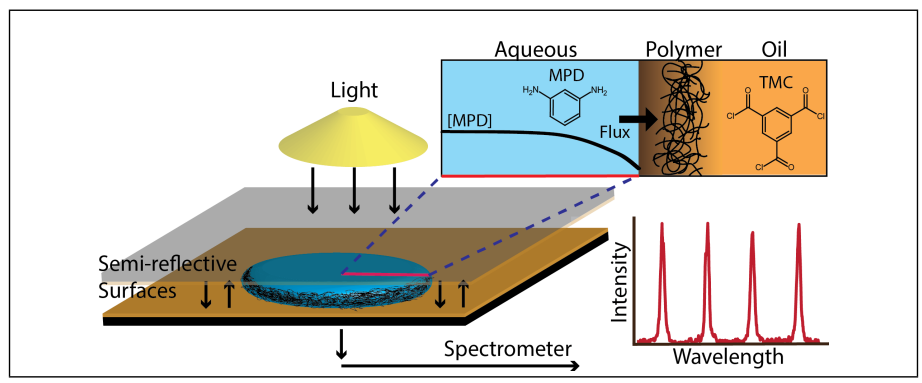

